\title{
Le numérique comme facilitateur de nouvelles interactions étudiantes entre apprenants experts : un cours en ligne pour enseigner l'anglais de spécialité
}

\author{
Using digital technology to facilitate educational interactions \\ between expert learners in an online course for English for \\ Specific Purposes
}

\section{La tecnología digital para facilitar las interacciones educativas en línea entre aprendices expertos: un curso en línea de inglés para fines específicos}

Mónica Fierro Porto, doctorante

Université de Paris, France

monicafierroporto@gmail.com

Lily Schofield, doctorante

Université de Paris, France

lilycschofield@gmail.com

RÉSUMÉ

Cet article présente le fonctionnement d'un premier dispositif de formation d'anglais en ligne sur la plateforme Moodle, intégrant les pratiques informelles des étudiants, à l'Université de Paris. Le choix d'utiliser des extraits de films dont l'intrigue est située dans le milieu professionnel financier (FASP) comme support pédagogique principal est à la fois adapté au public (première année de licence d'Économie-Gestion), et à l'intégration des pratiques informelles étudiantes en anglais au contexte formel du cours. Ainsi, par cette familiarité avec le contenu, les thématiques et les modalités d'interactions asynchrones en ligne, les étudiants deviennent apprenants experts et le format numérique favorise alors de nouvelles interactions. Nos résultats proviennent des données recueillies auprès des étudiants concernant leurs ressentis sur le dispositif et sur leur apprentissage. 
Mots-clés : formation à distance, apprentissage collectif/collaboratif, communication médiatisée par ordinateur (CMO), apprentissage informel, fiction à substrat professionnel (FASP), anglais de spécialité (ASP), apprenant expert

ABSTRACT

This article presents the first online English course on Moodle's learning management system at the University of Paris, which integrates students' informal practices. The use of extracts from films whose plot is set within the professional financial environment (FASP) as teaching material is adapted to students in the first year of an Economics and Management degree and allows us to integrate informal practices in the English course in a more formal context. This familiarity with the content, topics, and online asynchronous interactions means that students become expert learners, while the course's digital format encourages new interactions. Our results are based on students' data regarding their perception of the course and their language learning.

Keywords: online learning, collaborative learning, computer-mediated communication (CMC), informal learning, profession-based fiction (FASP), English for Specific Purposes (ESP), expert learner

\section{RESUMEN}

Este artículo presenta el funcionamiento de un primer dispositivo de enseñanza de inglés en línea que integra las prácticas informales de los estudiantes de la Universidad de París, utilizando la plataforma Moodle. La utilización de extractos de películas cuya trama representa un ámbito financiero profesional (FASP) como material pedagógico principal, se adapta tanto al público (primer año de licenciatura en Economía y Administración), como a la integración de prácticas informales en inglés en el contexto más formal del curso. Dada la familiaridad con el contenido, los temas y las modalidades de interacciones asincrónicas en línea, los estudiantes se convierten en aprendices expertos, el formato digital promoviendo así nuevas interacciones. Nuestros resultados provienen de datos recopilados acerca de las percepciones de los estudiantes sobre el dispositivo y sobre su aprendizaje de la lengua.

Palabras clave: formación a distancia, aprendizaje colaborativo, comunicación mediada por ordenador (CMO), aprendizaje informal, ficción con sustrato profesional (FASP), inglés con fines específicos (IFE), aprendiz experto

\section{Introduction}

L'enseignement des langues pour spécialistes d'autres disciplines (LanSAD) en France est une partie intégrante de la formation universitaire. L'enseignement de l'anglais comme langue vivante obligatoire est un choix fréquent des universités, influencé par la globalisation de cette langue comme outil de communication. L'hégémonie de l'anglais et l'accès démocratisé aux technologies facilitent le contact 
informel des apprenants avec la langue en tant qu'utilisateurs, et créent ainsi un écart entre la manière dont ils accèdent au savoir en autonomie et en salle de classe.

Les recherches en didactique des langues ont montré les nombreux avantages sur le développement langagier de l'exposition à la langue en dehors de la classe, notamment par le visionnage de séries et de films en anglais, avec ou sans sous-titres. II s'agit tout d'abord d'une activité répandue, pour laquelle le contenu est largement disponible : $90 \%$ des Européens disent regarder la télévision au moins une fois par semaine, près d'un tiers la regardent sur Internet (Commission Européenne, 2020). Ce visionnage améliorerait par ailleurs la maitrise linguistique et lexicale (Lindgren et Muñoz, 2013) en raison de l'exposition à une grande quantité d'outputs authentiques et parlés (Kuppens, 2010; Webb, 2015) ou de la répétition de mots peu fréquents (Webb et Rodgers, 2009a, 2009b), et favoriserait l'acquisition incidente de vocabulaire (Sockett, 2014; Peters et Webb, 2018), collocations (Gonzalez-Fernández et Schmitt, 2015), phrases verbales (Schmitt et Redwood, 2011), unités de langue ou " chunks " (Sockett, 2011; Kusyk et Sockett, 2012; Puimège et Peters, 2019).

Le dispositif intègre cette pratique au contexte formel d'un cours en ligne en utilisant des fictions à substrat professionnelles au format filmique (FASP). Ces œuvres, initialement des romans ou " thrillers spécialisés » (Petit, 1999), ont recours à « un milieu professionnel particulier non seulement comme cadre général de l'histoire mais aussi et surtout comme l'une des sources principales des ressorts de l'intrigue » (Petit, 2000, p. 173-174). Leurs apports spécifiques à l'enseignement de l'anglais de spécialité seront détaillés dans les sections suivantes.

L'intégration de ces ressources pourrait avoir un effet positif sur la motivation des apprenants et leurs représentations vis-à-vis de l'apprentissage de la langue. En effet, dans l'enseignement supérieur en France, les cours d'anglais LanSAD se font souvent en groupes de grande taille, limitant ainsi l'interaction, et leur contenu est parfois éloigné du domaine étudié. En effet, l'enseignement formel de l'anglais en France est souvent centré sur la culture anglo-saxonne travaillée à travers des œuvres classiques ou littéraires et est donc souvent " très loin de l'expérience de communication en anglais de la plupart des apprenants aujourd'hui » (Sockett et Kusyk, 2013).

Or, ce dispositif a pour but d'intégrer les pratiques informelles des étudiants au contexte formel de ce cours en ligne. L'apprentissage informel peut être défini comme résultant " d'activités quotidiennes liées au travail, à la vie de famille et aux loisirs » qui peut ou non être intentionnel, par opposition à l'apprentissage formel qui serait intentionnel et « généralement dispensé par des établissements d'enseignement ou de formation (...) et entraînant une certification " (Stevens et Shield, 2010, p. 12). En proposant la mise en ligne de l'enseignement de l'anglais en licence d'Économie-Gestion, l'Université de Paris a souhaité pallier cette divergence entre le temps consacré à l'apprentissage en contexte formel et en dehors. De même, cette solution réduit l'écart entre les pratiques d'enseignement en milieu universitaire et les activités réelles des étudiants qui accèdent à l'information en anglais informellement.

Notre étude s'appuie sur les recherches montrant les nombreux apports du numérique et du distanciel, notamment en ce qui concerne la communication asynchrone médiée par ordinateur et les opportunités d'apprentissage collaboratif. Elle tient également compte des recherches disciplinaires sur certains aspects du développement langagier en langue seconde, en anglais de spécialité et sur les supports envisagés pour son enseignement. L'enseignement à distance dans le supérieur n'a rien de novateur et notre dispositif tient compte de ses nombreux mérites tels que ses effets sur la motivation et l'autonomie, et la flexibilité (travail asynchrone au rythme de l'apprenant, tentatives multiples possibles en fonction des besoins ou préférences, gestion du temps). L'apport principal de notre dispositif réside dans l'intégration des pratiques informelles des étudiants au contexte formel de cet enseignement à distance : notre choix de ressources, de contenu et d'activités se base sur ce que les étudiants font habituellement en dehors des cours et met l'accent sur ce qu'ils apportent au cours, du fait de leur familiarité avec les éléments du 
dispositif. Ceci donne lieu à des interactions étudiantes inédites, favorisées et facilitées par le numérique, au sein desquelles les apprenants ont le rôle d'experts en herbe de leur domaine d'études et de connaisseurs des contenus proposés, qui sont liés à leurs propres pratiques. Les tâches proposées nécessitent de faire des recherches en ligne et de faire le lien avec des savoirs préalables ou des expériences personnelles afin que les étudiants deviennent transmetteurs des savoirs.

\section{Contexte et problématique}

La conception de ce dispositif d'enseignement en ligne s'inscrit dans la mise à distance des cours d'anglais en première année de licence d'Économie et Gestion pour l'année universitaire 2017-2018, dans l'environnement numérique d'apprentissage Moodle, et a été reconduite tous les ans depuis.

Le cours s'adresse à des étudiants de l'anglais LANSAD, plus précisément en anglais de spécialité (ASP), défini comme " la branche de l'anglistique qui traite de la langue, du discours et de la culture des communautés professionnelles et groupes sociaux spécialisés anglophones et de l'enseignement de cet objet » (Petit, 2002, p. 2-3). Ces contenus d'enseignement sont conçus en fonction des besoins des apprenants, souvent adultes ou étudiants du supérieur, d'un niveau intermédiaire ou avancé (DudleyEvans et St John, 1998, p. 4-5). L'enseignement se caractérise par une interaction entre les connaissances de la langue et du contenu disciplinaire, une orientation vers un objectif spécifique, des compétences et connaissances précises (en langue et culture) de la discipline universitaire ou des métiers du domaine, la possibilité d'accomplir des tâches « de la vie réelle ", ainsi que certaines contraintes institutionnelles telles que l'hétérogénéité des profils et niveaux, l'aspect obligatoire du cours influant la motivation, la taille importante des groupes et le temps de contact restreint (Sarré et Whyte, 2016). En effet, la connaissance d'un lexique étendu en langue étrangère serait étroitement liée à celle du domaine professionnel en soi, et ainsi primordiale à la réussite des études et pour la vie professionnelle de l'apprenant (Coxhead, 2017).

La conception de notre dispositif, ses principes et son format distanciel permettent de tenir compte de ces divers principes et de pallier les contraintes courantes en ASP. Nous développerons à la fois les avantages de l'enseignement à distance en apprentissage des langues, les apports supplémentaires de notre dispositif et de nos choix didactiques, ainsi que certaines limites rencontrées.

\section{Présentation du dispositif}

\section{Les fictions à substrat professionnel comme ressource pédagogique principale}

Les supports pédagogiques principaux de notre dispositif sont de courts extraits de FASP sous leur forme filmique, " utilisant un milieu professionnel particulier non seulement comme cadre général de l'histoire mais aussi et surtout comme l'une des sources principales des ressorts de l'intrigue " (Petit, 2000, p. 173-174). Les films et séries choisis (Suits, The Big Short, Margin Call, The Wizard of Lies, Rogue Trader) représentent différents métiers et reflètent le milieu de la banque et de la finance. L'un des apports principaux de ces fictions, justifiant leur utilisation en anglais de spécialité (Isani, 2011; Chapon, 2015), chez un public se destinant à une carrière dans ce domaine potentiellement à l'international, est la réduction de l'écart entre les études et le monde professionnel envisagé par les étudiants. Elles représentent, selon Le Cor (2001), une médiation entre l'étudiant et le milieu professionnel. Leur valeur documentaire, à la fois culturelle et linguistique, permet donc une familiarisation avec le lexique et la terminologie du discours professionnel en contexte (Chaplier, 2011, p. 66), ainsi qu'avec les éléments culturels liés au domaine (Petit, 2004, p. 7). Elles présentent également les échanges procéduraux propres 
aux métiers représentés tels que les règles d'adresse ou de politesse, ainsi que leur fonctionnement, à travers les rapports hiérarchiques ou rituels au sein de la communauté professionnelle (Chapon, 2010) et montrent des aspects plus informels, comme les échanges entre collègues. Ceci en fait un bon outil pour la découverte de la culture d'entreprise et de l'éthique professionnelle (Hardy, 2011, p. 54).

Ce dispositif se décline en trois cours de niveaux progressifs, dont deux sont suivis sur l'année par chaque étudiant en fonction du niveau auto-déclaré en début de semestre sur le questionnaire de placement (questions d'auto-évaluation selon les niveaux et descripteurs du CECRL, note obtenue en anglais au baccalauréat). Chaque cours se compose de neuf modules représentant deux heures de travail personnel par semaine et introduisant un nouveau concept économique ou financier ou une question d'ordre éthique ou moral traités dans des extraits pertinents des films (figure 1). Les passages sont donc choisis pour leur apport didactique, selon le niveau du cours, en fonction du lexique et des concepts économiques et financiers présentés (délocalisation d'entreprise, crédit immobilier, titre de créance collatéralisé, pyramide de Ponzi), ainsi que pour la réflexion d'ordre morale ou éthique qu'ils engendrent sur le fonctionnement du milieu professionnel et le comportement à adopter (entretien d'embauche, discours en entreprise, techniques de neutralisation).

Les cours des différents niveaux se distinguent en termes de progression par le nombre de séries ou de films travaillés (un épisode de série, un film, trois films), la longueur des extraits (de 30 secondes à 10 minutes), le nombre de documents vidéo par module (entre un et quatre), le nombre d'interlocuteurs par extrait, la complexité des concepts présentés et l'accès à la transcription des extraits. Toutes les activités fermées de compréhension sont des évaluations formatives pouvant être recommencées de manière illimitée.

\section{Figure 1}

Exemple de production écrite

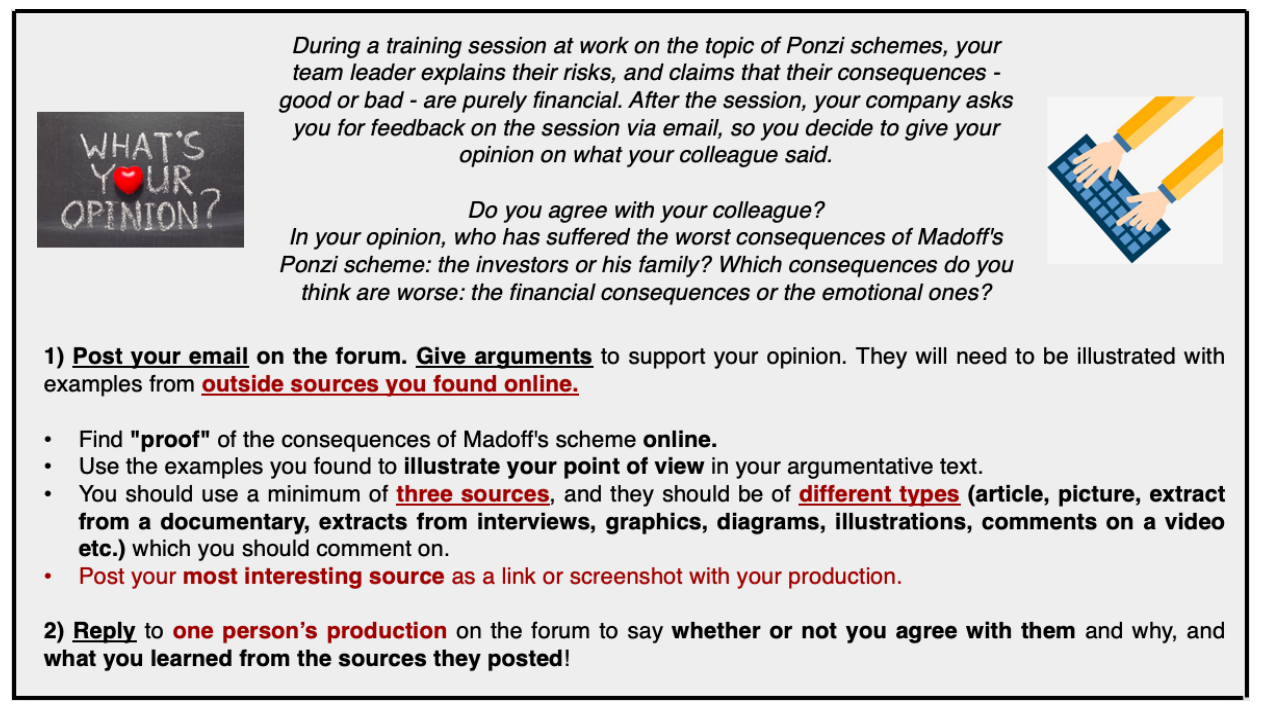

Les trois enseignants-tuteurs se présentent sous forme de vidéo lors de la première semaine de cours afin d'expliquer les consignes, les modalités d'évaluation et le contrat didactique, et d'inciter les étudiants à se présenter à leur tour. Chaque module débute par une activité de compréhension orale sur les extraits, suivie d'une tâche de production écrite ou orale, individuelle ou collective, sur le même sujet à partager sur le forum de discussion de la plateforme. Les étudiants sont encouragés à y inclure des informations collectées en ligne (figure 2) de diverses sources, à faire le lien avec leurs savoirs préalables et à partager et à comparer leur expérience personnelle sur ces sujets, les mettant en position d'apprenant expert. Enfin, 
la dernière activité renforce le lexique spécialisé présenté dans les extraits ou découlant du thème de la semaine sous forme de quiz ou de contribution à un glossaire collaboratif. Des documents complémentaires (articles de presse, vulgarisation, blogs, vidéos informatives ou ludiques) sont proposés en fin de module.

\section{Figure 2}

Exemple d'activité sur le lexique nécessitant le partage des pratiques informelles

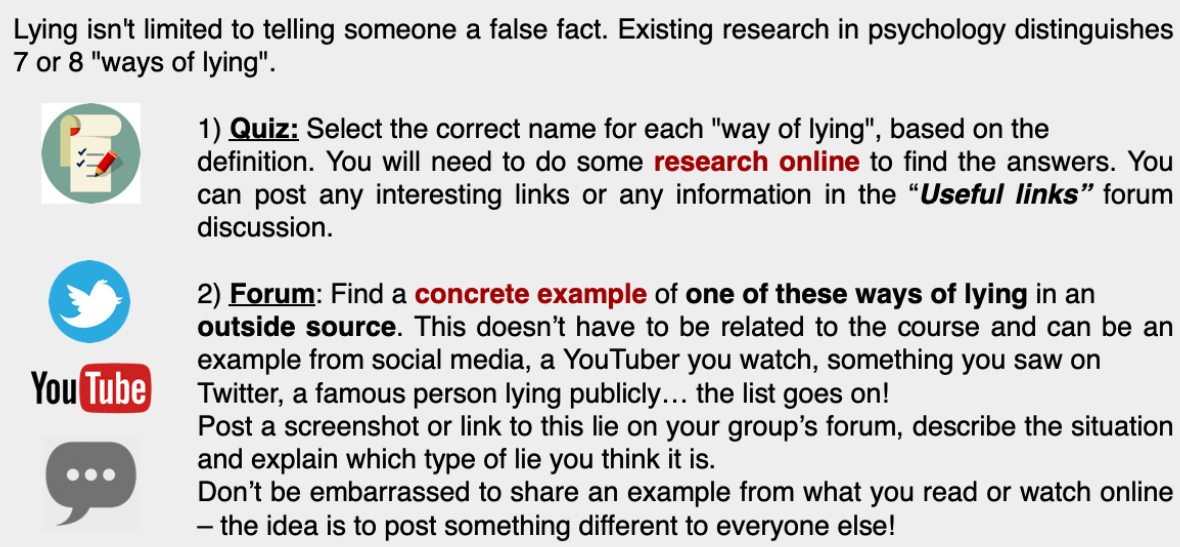

Dans notre dispositif, en tant qu'enseignants-tuteurs, nous nous sommes chargés de la conception, de l'instrumentalisation des contenus informels ${ }^{1}$, du développement des espaces numériques, de l'enseignement et de l'évaluation. L'implication majeure permet une vision globale de la pertinence de l'interface et des documents et tâches proposés dans l'apprentissage collaboratif et dans l'enseignement de l'anglais de spécialité.

\section{Intégration des pratiques informelles et motivation}

L'utilisation des FASP permet de réduire l'écart entre l'utilisation de l'anglais dans un contexte formel et celle des étudiants en dehors de leurs cours. En effet, le principe central de notre dispositif est l'intégration des pratiques informelles des apprenants au contexte formel de ce dispositif, notamment le visionnage de films en anglais, pratique courante chez le public universitaire en France (Sockett, 2011), ainsi que l'interaction en ligne, un ajout au Volume complémentaire du Cadre européen.

Dans le cadre de cette étude, l'interaction se fait via les forums de discussions de la plateforme, ce choix étant motivé par la familiarité des apprenants avec cette modalité de communication, puisque l'apprentissage serait favorisé lorsque l'activité proposée est associée à des sentiments positifs et provoque un intérêt chez l'apprenant (Krashen, 1982). Par ailleurs, la recherche atteste des effets positifs de la communication asynchrone écrite médiée par ordinateur sur la motivation et la volonté de communiquer (Maclntyre et al., 1998), ainsi que sur la production langagière à l'écrit. L'une des plus grandes affordances de l'ordinateur en termes de lecture et de communication est la " persistance » du texte sur l'écran (Blake, 2016: 129). II y aurait moins d'attention à la forme lors de la communication synchrone, en raison de la pression du temps (Stockwell, 2010), les structures syntaxiques seraient moins précises et plus proches de l'oral, mais plus variées (Sotillo, 2000). La grammaire serait moins complexe

\footnotetext{
${ }^{1}$ Des pratiques en ligne réalisées par les étudiants pour le loisir, sans qu'il y ait nécessairement une conscience sur l'impact de la pratique sur l'acquisition langagière Sockett (2011).
} 
par peur de prendre des risques, tandis que la communication asynchrone ralentirait le temps et favoriserait la prise de distance (Bruillard, 2003) ainsi que l'exposition des points de vue (Mangenot, 2002). Elle permettrait plus de temps de planification donc moins de frustration (Blake, 2016), donnerait lieu à une meilleure structure syntaxique et une meilleure cohésion (Stockwell, 2010). Les apprenants produiraient moins d'output qu'en synchrone, mais celui-ci serait plus juste syntaxiquement et plus complexe. Ce temps supplémentaire permettrait une meilleure focalisation sur la forme et le sens et moins de copie d'erreurs entre apprenants (Sotillo, 2000).

Ces observations semblent confirmées par les représentations de nos étudiants : lors du recueil des données via la passation des questionnaires et entretiens, les apprenants plus introvertis ont déclaré participer plus que lors d'un cours en présentiel, car ils ressentent moins d'angoisse liée au jugement des autres ou aux circonstances sources de stress telles que la situation d'examen. Le forum et les ressources encouragent la participation des apprenants-experts qui sont connaisseurs de leurs pratiques intégrées au cours, apportent leurs propres expériences, fournissent des documents issus de leurs recherches et sont experts en herbe de leur domaine d'études. Ceci crée une dynamique de groupe motivant davantage leurs interactions, quel que soit le niveau de langue et d'assurance des étudiants dans la langue étrangère.

\section{Nouvelles interactions entre apprenants experts de leur domaine}

Chaque semaine, les étudiants travaillent sur des tâches de compréhension (figure 3 ) et production (figures 4 et 5) pour lesquelles l'apprentissage est « autant basé sur l'appropriation d'informations que sur la création de contenu » (Papi, 2016a, p. 18). Ils doivent " parvenir à un résultat donné en fonction d'un problème à résoudre, d'une obligation à remplir, d'un but qu'on s'est fixé » (Conseil de l'Europe, 2001, p. 16). 
Figure 3

Exemple d'activité de compréhension orale

\begin{abstract}
Introducing a revolutionary idea
In this video you will see Lewis Ranieri, the man you saw at the end of the video in the last lesson. You will see him in action at work, first encouraging colleagues to increase profits by selling more bonds and securities. Then, you will see him at a meeting with some pension fund managers as he explains his revolutionary investment product idea: The Mortgage Backed Security or Private Label MBS. The pension fund managers need solid investments so that their clients can look forward to a secure retirement. Ranieri's product is stable and can earn a lot of interest.
\end{abstract}
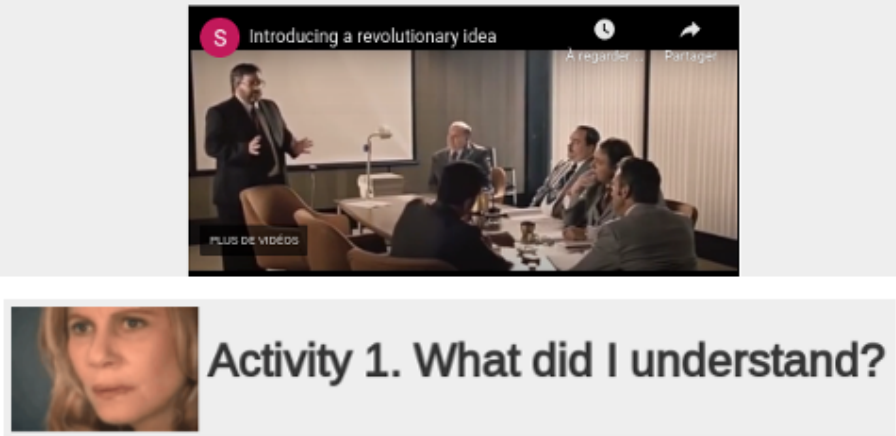

Which of the following statements is true?

\author{
Select one: \\ a. Bonds were considered a great investment. \\ b. People used to give them as gifts to family members. \\ c. Louis Ranieri invented the bond. \\ d. Bonds provided a fixed interest of 100 dollars.
}

Ces tâches permettent de travailler un concept financier et son lexique associé, une compétence professionnelle, ou de s'exprimer sur un aspect éthique des métiers représentés. Elles contiennent donc un manque d'information ou d'opinion rendant l'échange nécessaire (Prabhu, 1987). Elles ont été conçues dans un souci d'authenticité et de " sincérité » (Springer, 2010) afin, d'une part, d'encourager l'expression de réactions personnelles et d'assurer une réflexion critique sur les questions éthiques et la culture professionnelle et d'entreprise. D'autre part, en proposant des productions reflétant des situations pouvant se présenter dans le futur monde professionnel des étudiants, où l'usage de la langue ressemble " directement ou indirectement » à la façon dont elle serait utilisée dans la vie réelle (Ellis, 2003, p. 16), l'étudiant utilise les éléments appris dans un contexte qui fait sens pour lui, « relève de son univers actuel ou futur » (Nissen, 2011, p. 10) et il interagit avec le discours propre à son domaine d'études. La tâche est ainsi réaliste (Conseil de l'Europe, 2001, p. 121) et significative, représente un vrai enjeu pour l'apprenant et est familière dans son thème, genre textuel et interactions (Médioni, 2009, p. 8). 
Figure 4

Exemple d'activité de production orale

\section{Yُ Activity 2: Credit S.O.S. Broadcast

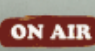

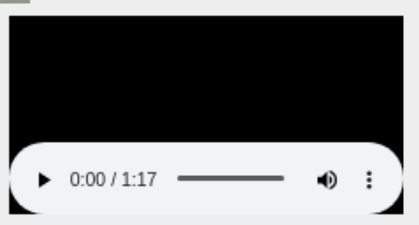

$\mathrm{Hi}$ there, this activity is called Credit S.O.S. Broadcast, and you are the invited expert!

You will read the article below about "The importance of credit reports and credit scores for building financial security". Then, you will make a one to two minutes sound file explaining the subject in everyday words. You will post it in the forum of your group, replying to the discussion "Credit S.O.S. Broadcast ». Use your name as the file name and deposit it for the others to listen to.

You can use your phone to record your voice, just make sure there's no noise around you so we can hear you well. If your file is too heavy and you can't upload it directly onto the forum, you can use a cloud-based file transfer service, like (WeTransfer, Google Drive or Dropbox), upload your video and then post the link to download it onto the forum.

Can't wait to hear you!

Reading for Credit S.O.S Broadcast

Ces productions, écrites ou orales, sont à poster sur le forum de discussion de chaque sous-groupe (2530 étudiants) et demandent aux apprenants de faire référence à leurs savoirs préalables et propres expériences. L'enseignement de ce dispositif est une conjonction « d'un système rendant publiques toutes les contributions, d'une pédagogie fondée sur des tâches pertinentes et d'étudiants acceptant de jouer le jeu de la mutualisation » de celles-ci et peut être caractérisé comme un apprentissage collectif (Mangenot, 2002).

\section{Figure 5}

Exemple d'activité de production écrite ou orale au choix

\section{So, what do you think?}

Based on what you've seen, do you think that it's easier or more difficult to get conned today than it was 20 years ago? Has the Internet played a positive or negative role in stopping fraudsters, cheats, cons or scammers? Are we better informed on how to not get caught out, or are there more risks than ever before?

Have you ever heard about a particular MLM, seen a video or online post about one, been invited to join one or known someone who has? Have you ever been the victim of another fraud or trap online? What is your personal experience of getting conned in 2020 ?

Write on the forum OR post an audio file to tell us what you think using concrete examples, things you've seen yourself online, or personal experiences to justify your opinion! Remember that you can post screenshots or links from outside sources. 
Cette approche par tâches appelant à une communication dans un environnement en ligne n'a rien de nouveau (Nissen, 2003; Mangenot et Penilla, 2009; Ollivier, 2012), mais notre dispositif se base par ailleurs sur des pratiques et modalités d'interactions familières pour les apprenants, de par leurs pratiques informelles et leurs études dans le domaine représenté. Ils sont mis en position d'apprenants experts qui trouvent les informations nécessaires à l'accomplissement des tâches par eux-mêmes. Les idées et productions sont mutualisées grâce à l'environnement numérique, donnant lieu à de nouvelles interactions et à une médiation entre étudiants (Chaplier, 2011) en tant qu'experts en herbe, et ces échanges ainsi que le retour du tuteur deviennent les ressources centrales du cours.

\section{Pratique}

\section{Évolution du rôle de l'enseignant}

Ces nouveaux contextes d'enseignement modelés par l'intégration du numérique ainsi que les défis actuels de l'enseignement supérieur font évoluer le rôle de l'enseignant, particulièrement dans le cas des formations à distance (Ferone, 2017). L'enseignant doit développer de nouvelles compétences et s'impliquer dans des enjeux pédagogiques en adoptant plutôt un rôle facilitateur et managérial dans ces espaces (Papi, 2016b). Dans le cadre des formations à distance, les interactions entre apprenants et tuteurs via le numérique supposent le développement de liens sociaux et l'adaptation à l'apprentissage collaboratif dans ces espaces virtuels, ce qui représente une évolution des interactions existantes.

Les étudiants, ou apprenants experts voient leurs représentations sur la langue et leur apprentissage évoluer, et parviennent à se considérer comme connaisseurs. La manière dont les étudiants utilisent la langue informellement en ligne a changé ces dernières années (Dressman et Sadler, 2020; Lee, 2019; Sundqvist, 2009) pour devenir un réel outil de communication. Des écarts se créent donc entre cette utilisation pratique, réelle et immédiate de la langue et son utilisation en salle de classe. L'intégration de la technologie et des pratiques informelles en salle de classe permettrait alors de syntoniser les deux contextes, plaçant l'enseignant dans un nouveau rôle de tuteur et de médiateur.

La nature des tâches proposées accentuerait également ce sentiment d'expertise chez les apprenants et le rôle de l'enseignant en tant que transmetteur du savoir se trouverait ainsi minimisé. En effet, le dispositif inverse « les rôles apprenant/enseignant en demandant que les étudiants soient, pour un temps donné, les fournisseurs des supports pédagogiques »(Chapon, 2011, p. 126). L'enseignant-tuteur guide les apprenants et ranime les discussions, ponctue le déroulement du cours via des réponses aux questions, des commentaires sur les échanges et du feedback sur les productions, tout en laissant la place centrale aux apprenants et aux savoirs qu'ils contribuent à édifier. C'est justement cette inversion de dynamique qui favoriserait davantage l'autonomie des étudiants.

\section{Résultats}

Nous nous appuyons ici sur les représentations des étudiants de la session 2017-2018, première année de fonctionnement du dispositif. Des analyses quantitatives et qualitatives ont été réalisées, grâce notamment au recueil de données via des questionnaires pré et post cours, à des entretiens en fin de formation préparés et conduits par les enseignants-tuteurs auprès d'un échantillon volontaire correspondant à $20 \%$ des étudiants ayant répondu aux questionnaires. Ces derniers comportaient de 26 à 32 questions fermées, à choix multiples, à échelles et ouvertes. Les aspects évalués concernent la représentation des étudiants sur la langue et leur apprentissage, l'apprentissage collaboratif, l'utilité de la 
plateforme et l'utilisation de FASP en cours de langue. Nous avons pris en compte uniquement les étudiants ayant réalisé tous les questionnaires, obtenant ainsi la participation de 102 étudiants sur la promotion de 300. La plupart des étudiants étaient âgés de 17 à 19 ans (voir tableau 1), en majorité des femmes et principalement de nationalité française $(98,7 \%)$.

\section{Tableau 1}

Âge et sexe des étudiants

\begin{tabular}{cccc}
\hline Sexe & \multicolumn{3}{c}{$\hat{\text { Age }}$} \\
\hline & $\mathbf{1 7 - 1 9}$ & $\mathbf{2 0 - 2 2}$ & $\mathbf{2 3 - 2 6}$ \\
Homme & $35,70 \%$ & $6,60 \%$ & $0,00 \%$ \\
Femme & $40,80 \%$ & $16,30 \%$ & $0,50 \%$ \\
\hline
\end{tabular}

Le niveau de langue prédominant déclaré lors du test d'auto-évaluation était le niveau B1 $(37,4 \%)$, suivi de B2 $(26,3 \%)$ et de A2 $(14,3 \%)^{2}$.

\section{Représentations sur la langue et ressentis sur le cours}

Les représentations sur l'apprentissage de la langue reflètent bien le statut d'utilisateurs de la langue des étudiants, rôle en accord avec celui d'acteur social prôné par le CECRL et l'approche actionnelle, qui encourage l'accomplissement de tâches significatives en langue cible avec une focalisation sur le sens plutôt que la forme. Afin de connaître les avis sur les compétences nécessaires pour avoir un bon niveau de langue, nous avons demandé ce que signifiait "être bon en anglais " pour les étudiants. Leurs réponses indiquent que la communication est plus importante que d'autres aspects associés à des interactions plus formelles telles que la maitrise de la grammaire (figure 6).

\section{Figure 6}

Représentations des étudiants sur la maitrise de la langue

\section{Que faut-il pour être bon en anglais ?}

\section{Échelle d'importance}

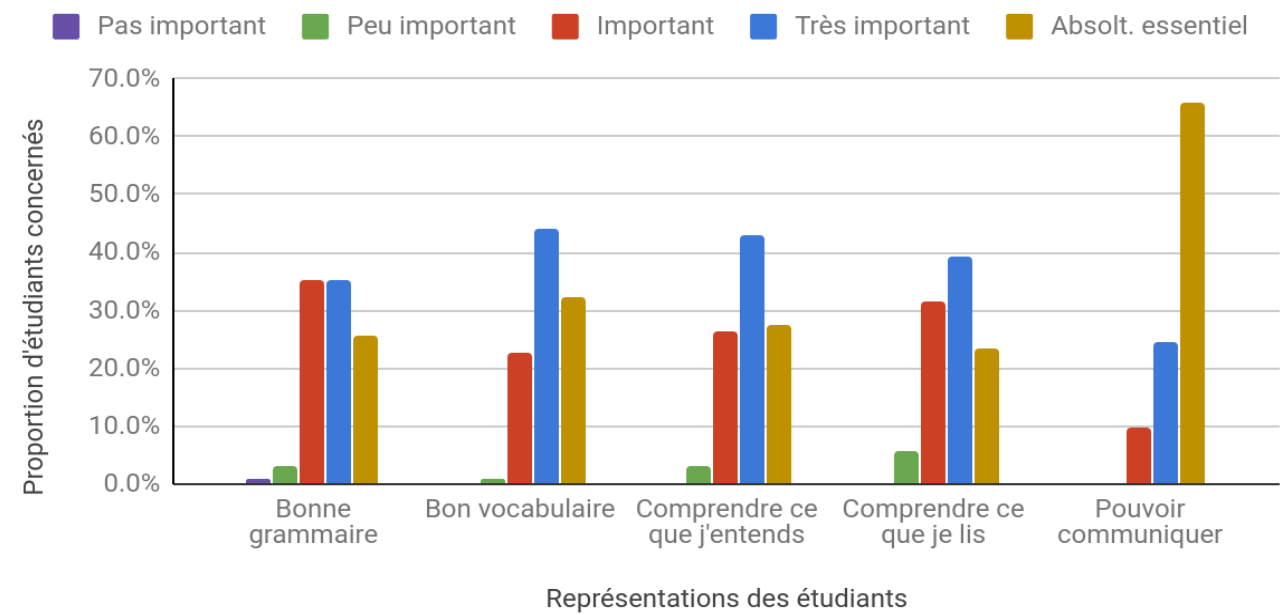

\footnotetext{
${ }^{2}$ Classement selon les niveaux du Cadre Européen Commun de Référence pour les Langues. A1 et A2 sont le niveau élémentaire, B1 et B2, le niveau indépendant, et $\mathrm{C} 1$ et $\mathrm{C} 2$, le niveau expérimenté.
} 
Les étudiants accordent une importance capitale à la possibilité de communiquer en anglais, que cela soit à l'écrit ou à l'oral. La différence entre les deux n'a pas été faite ici afin de connaître l'intérêt des étudiants pour l'acte de communication, peu importe le moyen. Il est intéressant d'observer que les pourcentages concernant la compréhension écrite et orale sont largement inférieurs à ceux de la communication. Ceci pourrait signifier que les étudiants font une différence entre les compétences langagières, mais que la maitrise de la langue se définit, pour eux, comme le fait de pouvoir communiquer et non pas d'avoir une maitrise parfaite des compétences.

\section{Figure 7}

Ressentis des étudiants sur le cours en ligne

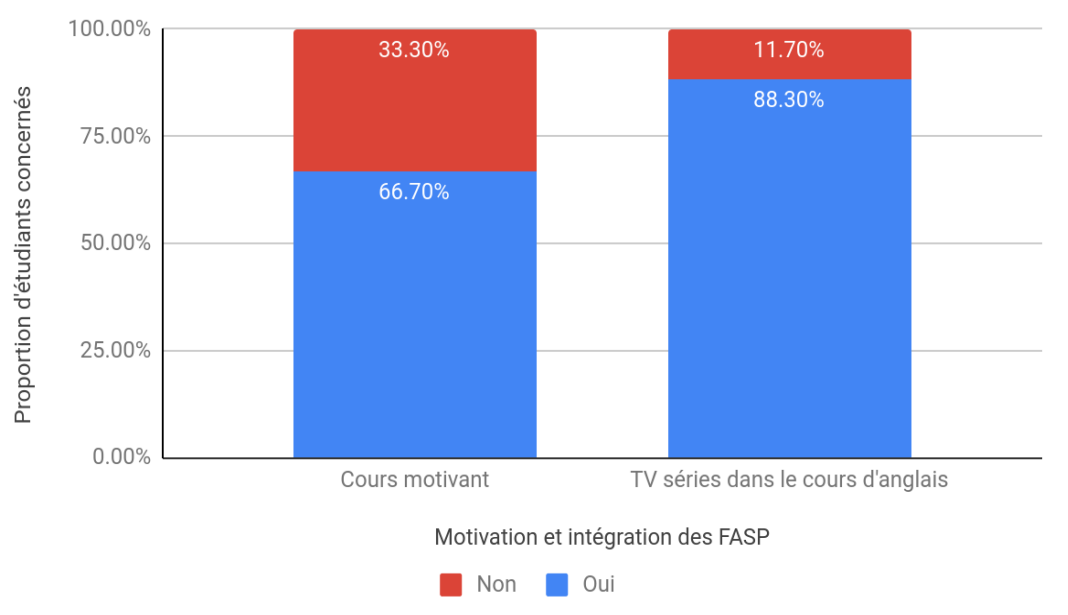

Leur ressenti sur l'utilisation des FASP dans le cours est positif : $88,30 \%$ des étudiants estiment que cette intégration était amusante et $66,7 \%$ considèrent le cours motivant (figure 7 ). Les étudiants semblent également avoir apprécié le format asynchrone du cours, surtout en termes de gestion du temps et de la flexibilité possible concernant le lieu d'apprentissage. 
Figure 8

Ressentis des étudiants sur le cours d'anglais en ligne

Le cours a servi pour:

Degré d'utilité

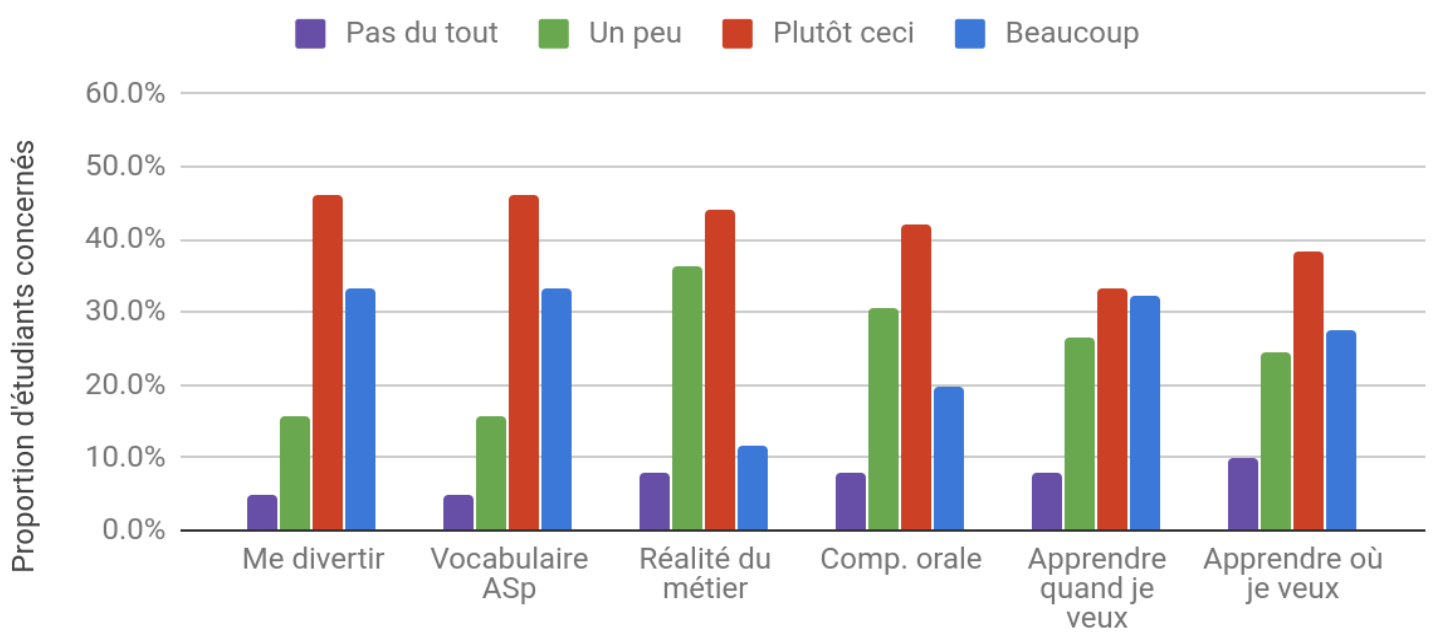

Aspects favorisés par le cours

Les étudiants considèrent avoir appris du vocabulaire de spécialité et ce ressenti est autant présent que leur sentiment de s'être divertis lors du cours. Ceci pourrait être un indicateur de l'intérêt des étudiants pour l'apprentissage du vocabulaire spécialisé utile à leur domaine d'études, puisque le cours met l'accent sur cette acquisition. Les étudiants ont aussi affirmé que les contenus leur ont permis de découvrir des situations propres à leur future réalité professionnelle, ce qui montre la possibilité de travailler des aspects culturels spécifiques avec les FASP (figure 8).

\section{Utilisation de l'interface et tutorat}

Le caractère distanciel a malheureusement généré un sentiment d'isolement chez une partie des étudiants. En effet, seulement 20,59 \% d'entre eux ne se sont pas sentis isolés par rapport à leurs pairs (figure 9). Ceci pourrait être une conséquence négative du manque d'interaction synchrone et du fait que les étudiants ne se connaissaient pas tous nécessairement ${ }^{3}$, rendant le tissage de liens entre eux plus difficile.

\footnotetext{
${ }^{3}$ Les étudiants sont répartis en groupes de travaux dirigés lors de leurs cours en présentiel, et ces groupes sont différents de ceux du cours d'anglais en ligne.
} 


\section{Figure 9}

Sentiment d'isolement des apprenants par rapport à l'interaction avec les pairs

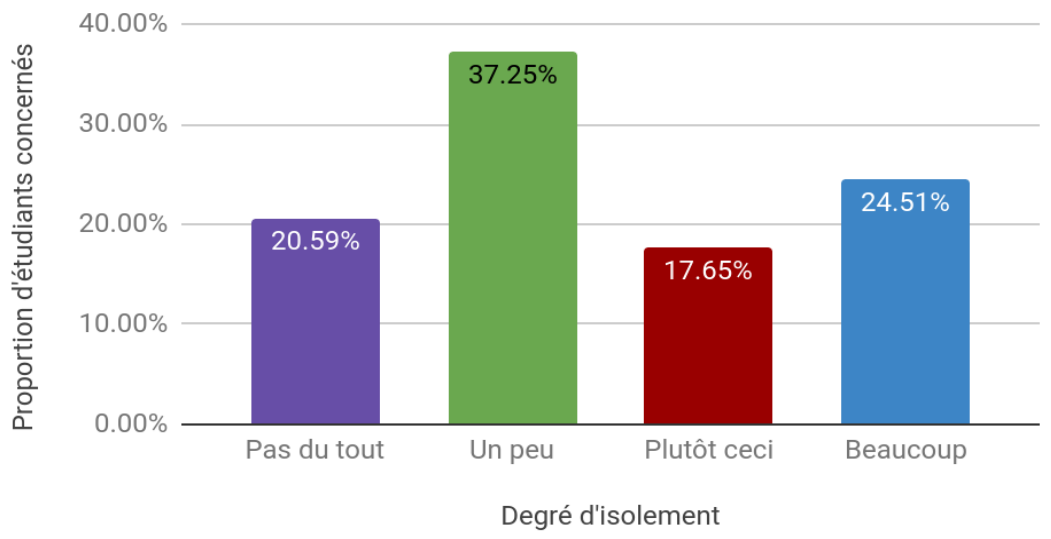

Quant à la plateforme et à la maitrise technologique, les étudiants ont trouvé que Moodle était plutôt facile d'utilisation et d'accès, et peu d'entre eux ont eu des difficultés technologiques majeures leur empêchant de réaliser les activités (figure 10).

\section{Figure 10}

Ressentis des étudiants sur le fonctionnement de la plateforme Moodle

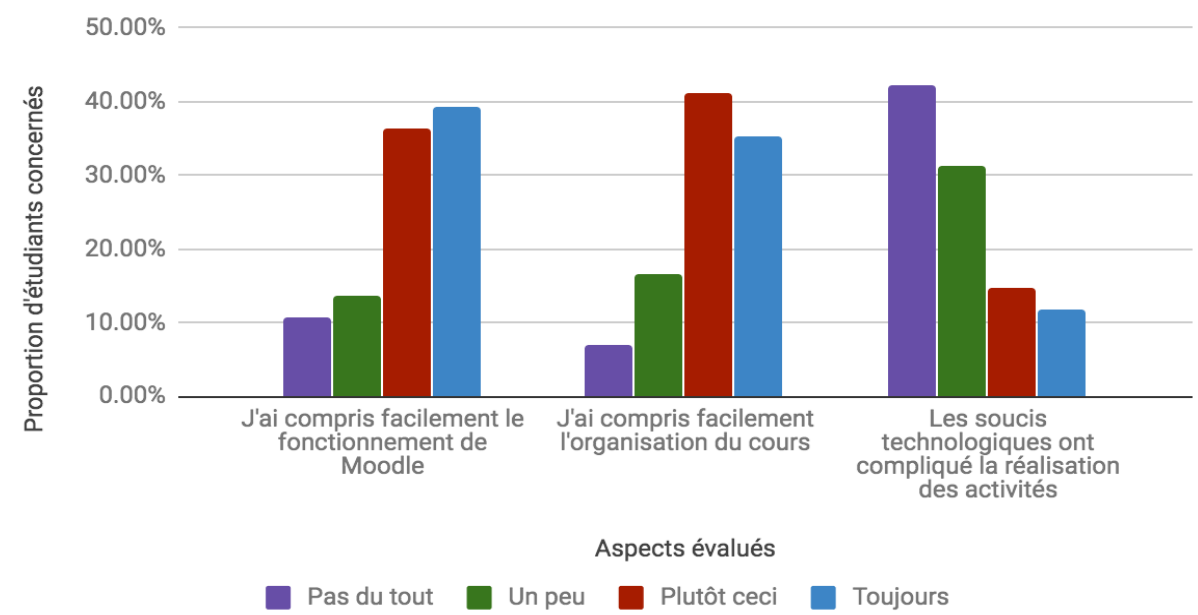

En effet, $84 \%$ des étudiants déclarent avoir facilement compris le fonctionnement du cours et ne pas avoir eu de difficultés pour accéder aux contenus et aux activités. Cependant, certains étudiants (10\%) ont eu des difficultés liées à la maitrise numérique, s'agissant souvent de dysfonctionnements liés à l'ergonomie de la plateforme, au temps de chargement avec une connexion Internet de puissance instable ou à l'utilisation des services de stockage et de partage en ligne pour des fichiers trop lourds ne pouvant être hébergés directement.

En termes d'apprentissage collaboratif, la fonctionnalité de forum a été privilégiée par les enseignantstuteurs (par rapport à l'atelier, les questionnaires ou le dépôt privé de devoirs), puisqu'il s'agit de l'espace où les étudiants partagent leurs productions et interagissent avec leurs pairs. Le forum permettait aux apprenants de publier ces productions de manière publique, facilitant l'apprentissage par les pairs. En effet, les étudiants utilisaient le forum pour voir ce que leurs camarades faisaient et pour se comparer afin de vérifier leur compréhension de la consigne et d'apprendre grâce aux autres. 


\section{Figure 11}

Ressentis des étudiants sur l'utilité du forum

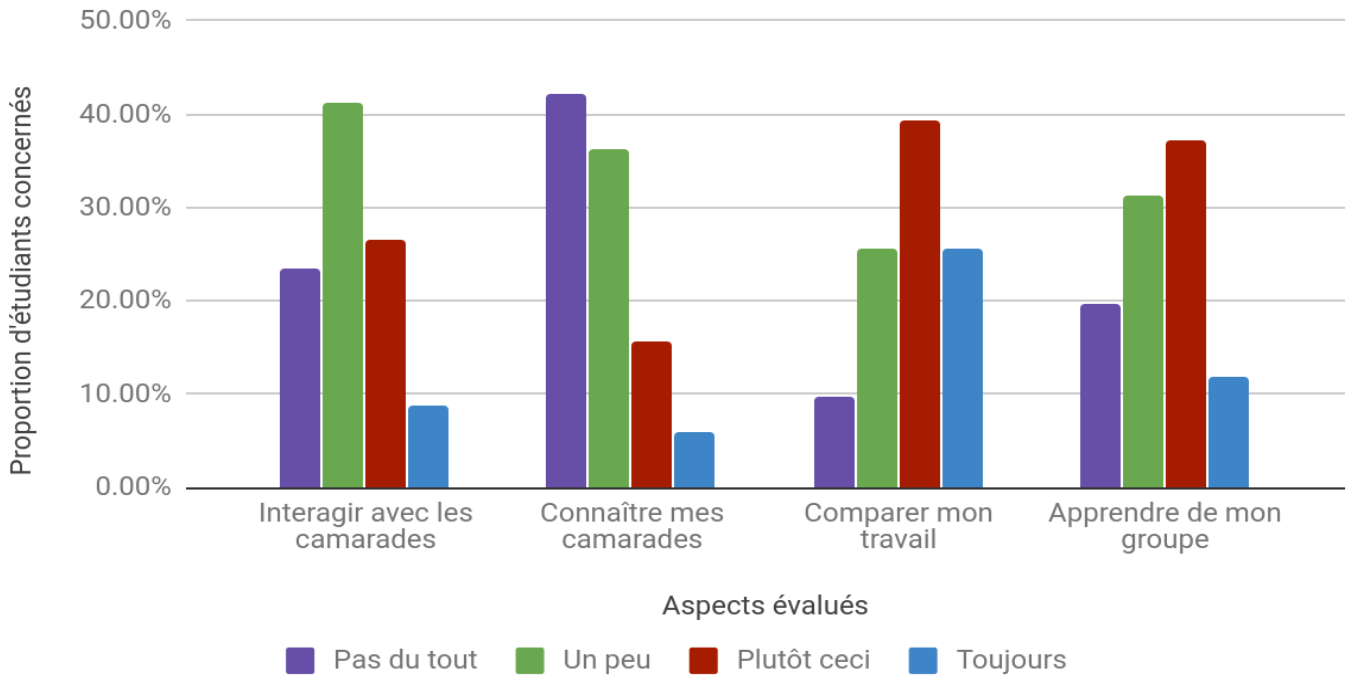

Le forum donnait également aux étudiants les outils pour interagir de manière asynchrone avec leurs pairs et leur tuteur. Ils n'ont malheureusement pas utilisé cet espace pour interagir entre eux de manière plus informelle. Le forum s'est donc avéré davantage utile pour l'apprentissage par les pairs que pour les interactions spontanées. Les étudiants ont surtout pu apprendre de leur groupe et comparer leur travail avec ceux des autres, mais $23,5 \%$ d'entre eux affirment ne pas avoir du tout interagi avec leurs camarades (figure 11). Le ressenti de manque d'interaction est accentué par le manque de possibilités de communiquer de manière synchrone et en face à face, modalités auxquelles les étudiants sont habitués en cours de langue, comme l'illustrent ces témoignages d'étudiants : "The oral activities. It was a little "strange" to speak alone and not to interact with other people »; " I don't like the fact that we have to use the forum to talk to our tutor » ou « We cannot interact very much with the students and we cannot speak English orally ».

Concernant le tutorat, les étudiants semblent avoir pu facilement et rapidement prendre contact avec les tuteurs et avoir obtenu des retours rassurants (93\%) et pertinents ( $87 \%$ ). Ces pourcentages concernent les trois enseignants-tuteurs impliqués dans l'enseignement du cours durant cette année. Les ressentis des étudiants sur leur accompagnement étant très similaires, ils ont été ici regroupés (figure 12). 


\section{Figure 12}

Ressentis des étudiants sur l'accompagnement

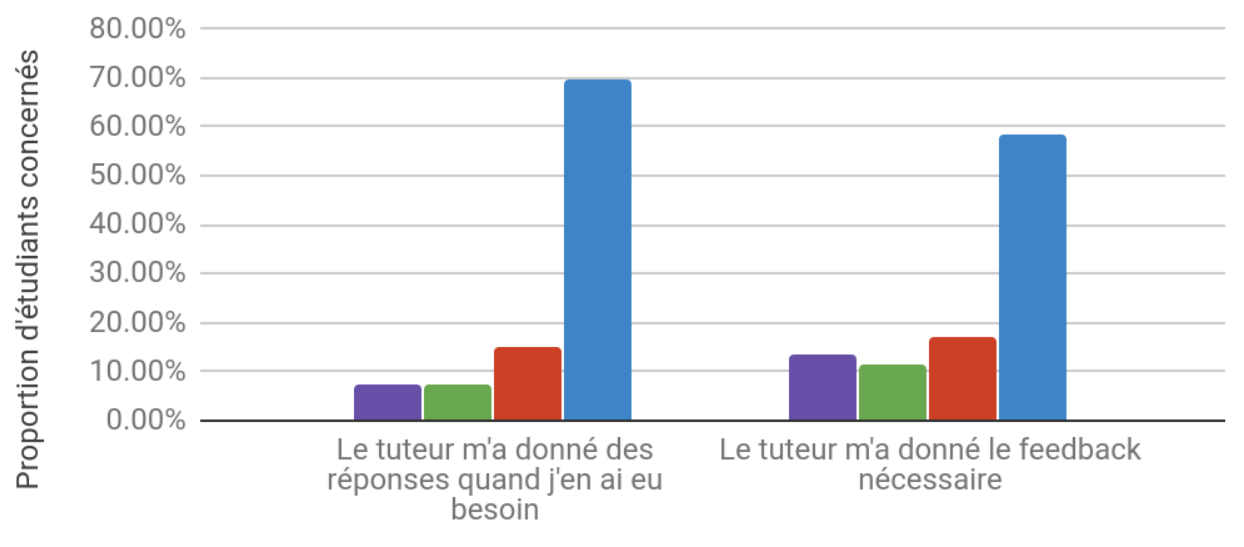

Acompagnement

Pas du tout $\square$ Un peu $\square$ Plutôt ceci $\square$ Beaucoup

Ces résultats très positifs par rapport à l'accompagnement peuvent être interprétés, d'une part, comme un effet du dispositif, dans le sens où ce dernier tient compte des particularités du distanciel et insiste sur les missions spécifiques de l'enseignant-tuteur. En effet, un cahier de charges du tuteur DIAL est présenté en début d'année afin de fournir un guide sur la réactivité attendue, la gestion de modules, l'évaluation et d'autres aspects liés à l'accompagnement (envoi de rappels, registre décontracté, veille et mise à jour de ressources). D'autre part, ces résultats pourraient être dus au degré d'investissement des enseignantstuteurs qui ont su se rendre disponibles à des horaires flexibles et répondre aux besoins des étudiants de manière pertinente.

Les réponses aux questionnaires peuvent être considérées comme l'illustration des critères expliquant la réussite du cours, comme le montrent les paroles d'étudiants compilées au tableau 2.

\section{Tableau 2}

Témoignages des étudiants sur le cours en ligne

\begin{tabular}{|c|c|c|}
\hline $\begin{array}{c}\text { Apprentissage par les } \\
\text { pairs }\end{array}$ & Stratégies d'apprentissage & Accompagnement \\
\hline $\begin{array}{c}\text { "What I like the most, see } \\
\text { what the others [sic] students } \\
\text { thinks [sic] of my } \\
\text { homework. " }\end{array}$ & $\begin{array}{c}\text { "The fact that we were learning } \\
\text { English on line [sic] was fun and } \\
\text { allowed us to have more liberties } \\
\text { about our work and how we wanted } \\
\text { to do it. » }\end{array}$ & $\begin{array}{c}\text { «I have contacted the tutor several } \\
\text { times and I have always had a very } \\
\text { quick answer and that helped me } \\
\text { a lot. » }\end{array}$ \\
\hline $\begin{array}{l}\text { " Everyone can participate } \\
\text { and be corrected by others. » }\end{array}$ & $\begin{array}{l}\text { "It was nice to be able to work my } \\
\text { English alone and at any time. I } \\
\text { was able to really concentrate and } \\
\text { progress, then post my work on the } \\
\text { forum and discover the work of the } \\
\text { other students in my group. " }\end{array}$ & $\begin{array}{l}\text { "The answers of my questions [sic] } \\
\text { were very quick and well explained. » }\end{array}$ \\
\hline $\begin{array}{l}\text { "Saw the activities of the } \\
\text { others on the forum, it was } \\
\text { really funny to see each } \\
\text { others [sic]. " }\end{array}$ & $\begin{array}{l}\text { "I learn English calmly at home } \\
\text { that allows me to take my time to } \\
\text { assimilate things and understand } \\
\text { them. " }\end{array}$ & $\begin{array}{c}\text { « Our tutor was always present when } \\
\text { needed. » }\end{array}$ \\
\hline
\end{tabular}




\section{Discussion}

La nouveauté de ce dispositif pour l'enseignement numérique de l'Université de Paris réside dans l'intégration des pratiques informelles des étudiants et la collaboration en ligne. En effet, la plateforme Moodle est utilisée depuis des années comme support des cours en présentiel, mais jamais des FASP n'avaient été utilisées pour enseigner l'anglais de spécialité. De plus, les cours de langue à l'Université étant jusque-là tous au format présentiel, la mise en place de notre dispositif a impliqué la création d'une proposition didactique pour le développement de cours entièrement à distance pour la Faculté d'ÉconomieGestion. II s'agit ainsi d'une première Didactique de l'Informel de l'Anglais en Ligne (DIAL) créée et développée dans le cadre d'une recherche-action au sein de l'Université de Paris. Cette innovation a permis d'établir une approche systématique pour la pérennisation des cours d'ASP en Économie-Gestion, en fonctionnement depuis 2017, mais aussi la création d'autres cours d'anglais dans d'autres spécialités, par exemple pour la Faculté de mathématiques.

Nos efforts en tant qu'enseignant-tuteurs se sont concentrés sur l'exploitation des fonctionnalités de l'interface permettant le travail collaboratif et l'apprentissage par les pairs, tout en promouvant le développement de l'autonomie de l'apprenant. Les résultats montrent que le choix des FASP a été pertinent et que leur instrumentalisation a eu un impact positif sur les ressentis des étudiants par rapport au cours. L'intégration des FASP aux cours formels a motivé le travail des étudiants sur la plateforme et cette instrumentalisation a facilité la mise en place d'activités générant chez la majorité des apprenants le sentiment d'avoir acquis du vocabulaire de spécialité. Ces résultats représentent déjà un succès dans l'enseignement de l'ASP à l'Université de Paris, car non seulement les ressentis des étudiants sur leur apprentissage de l'anglais de spécialité sont positifs, mais la réalité des pratiques informelles des étudiants a été intégrée au contexte formel, réduisant l'écart entre les deux univers. Les affirmations des apprenants nous permettent de supposer une perméabilité entre les deux, de manière à ce qu'ils puissent être conscients de l'utilité de leurs pratiques en dehors des cours dans l'acquisition incidente de la langue et donc éventuellement être plus attentifs à l'input reçu, mais aussi qu'ils puissent trouver un intérêt particulier dans des cours qui reflètent leurs intérêts, possiblement améliorer leur maitrise, augmenter leur motivation et ainsi réduire le risque de décrochage.

L'utilisation de l'espace numérique d'apprentissage Moodle s'est avérée utile pour faciliter le travail collaboratif et l'apprentissage entre apprenants experts. Pourtant, les fonctionnalités du forum permettant aux étudiants d'interagir entre eux ont été sous-exploitées, ce qui n'a pas motivé l'interaction spontanée entre étudiants. Ces derniers ont malgré tout réussi à partager leurs productions, à évaluer leurs pairs et à apprendre grâce à ces échanges. Le forum semble avoir été privilégié en raison de sa ressemblance aux chats et blogs sur Internet (commentaires publics, asynchrones et décontractés), permettant aux étudiants d'avoir des interactions similaires à leurs pratiques informelles. II a facilité les échanges, a hébergé des activités impliquant la mobilisation des compétences linguistiques et transversales (lexicales, références et connaissances culturelles, médiation) et a démystifié le partage au sein des groupes des productions écrites et orales, puisqu'il s'agissait d'un environnement bienveillant de co-construction et de mutualisation des savoirs.

La recherche a démontré que le succès dépend du système (objectifs, plateforme, fonctionnalités et ressources), de la chronologie du dispositif (rythme de travail et contrat didactique) et de l'accompagnement (tutorat) (Mangenot, 2002, p. 109). Nous considérons que la réussite de ces échanges pourrait en grande partie être expliquée par la clarté des règles de savoir-vivre propres aux échanges virtuels ainsi que par la médiation par l'enseignant-tuteur (Glikman, 2011). Nous encourageons une atmosphère informelle et détendue, ainsi qu'un feedback bienveillant entre étudiants et de la part des tuteurs, correspondant à l'approche que nous appelons DIAL. L'enseignant-tuteur a un rôle moins visible dans ce type de contexte, mais tout aussi important qu'il ne l'est en salle de classe : il incite à la 
participation, donne des retours pertinents et montre l'exemple de ce qui est attendu. II rassure également sur les aspects du format distanciel auxquels les étudiants ne sont pas forcément habitués tels que la maitrise de la plateforme ou le travail en autonomie. Enfin, il est chargé de l'évaluation, centrée sur le contenu et sur les échanges plutôt que sur la forme. Toutes ces dispositions de fonctionnement du cours sont présentées aux étudiants dès le début du cours afin de les pousser à produire davantage en langue cible. Les étudiants enquêtés ont expliqué que l'approche décontractée des enseignants-tuteurs, leurs retours encourageants et la focalisation des discussions sur les contenus des productions les avaient encouragés à partager et poster davantage sur le forum. Cependant, comme nous avons pu l'observer dans les résultats, les étudiants n'ont pas utilisé le forum pour communiquer entre eux, mais plutôt pour s'entraider grâce à la mise à disposition des travaux de manière publique. De plus, malgré les possibilités d'interaction proposées par l'interface, celles-ci impliquent plutôt des échanges écrits et certains étudiants ont ressenti un manque d'interaction orale. Les échanges oraux synchrones, souvent propres au contexte présentiel, sont toujours privilégiés par les étudiants de nature participative et extravertie. Une option complémentaire a ainsi été proposée à ces étudiants, mettant à disposition des ateliers de langue pour discuter en anglais avec des étudiants anglophones.

\section{Conclusion}

Le dispositif a été créé pour répondre à des besoins identifiés de l'Université tels que le désengorgement des salles de cours et l'intégration du numérique et des pratiques informelles des étudiants. Les ressentis exprimés ici permettent de tirer certaines conclusions sur l'efficacité du dispositif. D'une part, les étudiants ont apprécié l'introduction des FASP dans la formation et, d'autre part, leurs témoignages laissent supposer que cela aurait pu avoir un effet sur l'acquisition du vocabulaire de spécialité. En effet, les ressentis positifs des étudiants ayant suivi ce dispositif de formation durant l'année universitaire 20172018 peuvent être considérés comme des indices de l'utilité de l'intégration des FASP au contexte formel, en termes de motivation et d'autonomisation des apprenants. Le dispositif a veillé à surmonter le défi du décrochage et de l'isolement, souvent associés aux cours en ligne. Les étudiants ont effectivement témoigné positivement des interactions sur le forum qui leur ont permis de partager des informations et des expériences personnelles avec leurs pairs en tant qu'apprenants-experts, et qui les ont également motivés à mener à bien les tâches proposées.

En termes d'apprentissage, les étudiants considèrent avoir acquis du vocabulaire de spécialité en anglais (figure 4). Cet apprentissage pourrait être associé à l'utilisation des FASP, puisqu'il s'agit des ressources privilégiées en cours et que les supports pédagogiques complémentaires (documents écrits, vidéos, images, liens vers d'autres sites, etc.) ont été également choisis en fonction des FASP proposées. De même, les témoignages des étudiants mettent en évidence la mobilisation de compétences langagières, culturelles et de médiation, et la co-construction de savoirs lors de tâches de production individuelles et collectives. En effet, les étudiants affirment avoir développé des stratégies d'apprentissage (tableau 2) telles que la gestion du temps, de leur apprentissage à leur propre rythme et un esprit critique leur permettant d'apprendre des autres. Ceci semble avoir été facilité par le numérique, qui met à disposition des fonctionnalités telles que le forum et l'atelier, qui facilitent les interactions à propos des tâches et des contenus du cours et incitent la participation de différents profils d'apprenants (âge, études précédentes donc connaissances préalables variées, niveau de langue). Ces interactions favoriseraient le développement d'un esprit analytique sur leur propre performance et celle des autres, menant à des réflexions contribuant aux apprentissages. Cependant, le dispositif se base sur les ressentis : les mesures de cette étude ne permettent pas d'affirmer une progression langagière en tant que telle, mais plutôt une amélioration de l'assurance des étudiants vis-à-vis de l'anglais. 
Concernant l'accompagnement de l'enseignant-tuteur, d'une part, les apprenants affirment qu'il était opportun et pertinent, trouvant le guide dont ils avaient besoin lors de la réalisation des tâches et du partage sur la plateforme. D'autre part, nous avons constaté la richesse de notre mission, qui prend une tout autre dimension lorsque les interactions ont lieu virtuellement. En effet, l'accompagnement du tuteur va au-delà de l'enseignement de la langue; il comprend la manipulation de l'outil et l'optimisation de son exploitation, la gestion d'éventuels problèmes liés à l'utilisation de la technologie de la part des apprenants, le maintien d'un lien affectif et, surtout, une présence constante à distance. Concernant ce dernier aspect, nous aimerions ici emprunter les mots d'une étudiante qui affirme, lors d'un entretien post-cours, pour une question sur la présence et l'accompagnement de l'enseignant-tuteur, qu'il était « impossible pour un tuteur d'un cours en ligne d'être absent ", faisant un parallèle avec d'éventuelles absences d'enseignants qui génèrent l'annulation de cours présentiels. Cette phrase est l'évidence non seulement de l'importance de l'action et de l'interaction du tuteur et du sentiment de soutien ressenti par les étudiants, mais aussi de leur engagement dans un dispositif où les contraintes temporaires ou géographiques n'existent plus.

Un certain nombre de limites dans le développement du dispositif restent à résoudre : les lacunes en maitrise technologique de certains étudiants et des tuteurs, certains problèmes de repérage, de compréhension de consignes ou de partages de travaux liés à l'ergonomie de la plateforme. La production orale est un sujet controversé auprès des étudiants, car les interactions ne se déroulent pas de la même manière qu'en format présentiel. II est nécessaire de s'adapter à des interactions orales asynchrones qui ne sont pas toujours aussi spontanées qu'en salle de classe et qui peuvent générer un sentiment d'isolement. En effet, les ressentis des étudiants révèlent que les canaux de communication choisis n'ont pas permis de mettre en œuvre tous les processus liés à l'interaction. Les étudiants ont apprécié les activités de production orale proposées par le dispositif et les considèrent comme "suffisantes » en termes de production et non d'interaction, mais affirment être en manque d'interaction orale synchrone et suggèrent même des modifications pour interagir avec aisance dans le dispositif (moments de chats synchrones, format live avec des horaires précis par semaine, ateliers de conversation).

La réussite de ce dispositif est mesurée selon les ressentis des étudiants et leur performance pendant l'année, mais doit également être perçue comme un ensemble où tous les choix (plateforme, ressources, règles, accompagnement) ont joué un rôle capital. Selon ces résultats et gardant en vue les améliorations nécessaires en termes d'interaction, les cours en ligne peuvent représenter une alternative efficace pour répondre aux besoins des étudiants et de I'Université. Ils donnent lieu à une participation élevée et s'avèrent une bonne solution contre l'absentéisme souvent important en première année de licence. Les ressentis des étudiants concernant l'intégration des FASP, le format distanciel et l'acquisition du vocabulaire de spécialité illustrent bien une réussite dans la volonté de réduire l'écart entre formel et informel. Ces cours sont toujours utilisés en première année de licence; nous formons de nouveaux tuteurs et le programme évolue chaque année, à la suite des retours des étudiants.

\section{Liste de références}

Blake, R. (2016). Technology and the four skills. Language Learning and Technology, 20(2), $129-142$. http://doi.org/10125/44465

Bruillard, É. (2003). Lorsque la distance favorise le temps et la réflexion. Medialog, 46, 53-54.

Chaplier, C. (2011). Le support filmique comme outil de médiation entre enseignant et étudiants LANSAD-sciences. Recherche et pratiques pédagogiques en langues de spécialité - Cahiers de l'APLIUT, 30(3), 61-74. https://doi.org/10.4000/apliut.1654

Chapon, S. (2010). Vera Drake et le Times: L'adstrat juridique cinématographique comme soutien pédagogique du document authentique. ILCEA, 12. https://doi.org/10.4000/ilcea.458 
Chapon, S. (2011). Vous avez le droit de garder le silence : Un scénario pédagogique pour faire parler les étudiants de droit. Recherche et pratiques pédagogiques en langues de spécialité - Cahiers de l'APLIUT, 30(1), 117-128. https://doi.org/10.4000/apliut.477

Chapon, S. (2015). Fiction à substrat professionnel télévisuel comme voie d'accès à l'enseignement/apprentissage de l'anglais juridique [thèse de doctorat, Université Grenoble-Alpes].

Commission Européenne (2020). Les habitudes médiatiques dans l'Union européenne. Office des publications de l'Union européenne. https://op.europa.eu/en/publication-detail/-/publication/c2fb9fad-db78-11ea-adf7-01aa75ed71a1/ language-fr/format-PDF

Conseil de l'Europe. (2001). Cadre Européen Commun de Référence pour les Langues: Apprendre, Enseigner, Évaluer (Conseil de l'Europe). https://rm.coe.int/16802fc3a8

Coxhead, A. (2017). Vocabulary and English for Specific Purposes Research: Quantitative and Qualitative Perspectives. Routledge. https://doi.org/10.4324/9781315146478

Dressman, M. et Sadler, R. W. (s. d.). (2020). The Handbook of Informal Language Learning. Wiley-Blackwell.

Dudley-Evans, T. et St John, M. J. (1998). Developments in English for Specific Purposes: A Multi-Disciplinary Approach. Cambridge University Press.

Ellis, R. (2003). Task-based Language Learning and Teaching. Oxford University Press.

Ferone, G. (2017). Effets perçus de l'engagement en formation à distance sur les pratiques et les compétences des enseignants du supérieur. Distances et médiations des savoirs. Distance and Mediation of Knowledge, 18, Article 18. https:/doi.org/10.4000/dms. 1890

Glikman, V. (2011). Tuteur à distance : Une fonction, un métier, une identité? Dans B. De Lievre, C. Depover, A. Jaillet, D. Peraya et J.-J. Quintin (dir.). Le tutorat en formation à distance (p. 137-158). De Boeck Supérieur.

González Fernández, B. et Schmitt, N. (2015). How much collocation knowledge do L2 learners have?: The effects of frequency and amount of exposure. ITL - International Journal of Applied Linguistics, 166(1). https://doi.org/10.1075/itl.166.1.03fer

Hardy, M. (2011). FASP et culture d'entreprise: Les représentations fictionnelles, passerelles entre la réalité et les perceptions des apprenants? Recherche et pratiques pédagogiques en langues de spécialité - Cahiers de l'APLIUT, 30(2), 46-64. https://doi.org/10.4000/apliut.1597

Isani, S. (2011). Developing Professional Cultural Competence through the Multi-layered Cultural Substrata of FASP: English for Legal Purposes and M. R. Hall's The Coroner. Recherche et pratiques pédagogiques en langues de spécialité Cahiers de I'APLIUT, 30(2), 29-45. https://doi.org/10.4000/apliut.1497

Krashen, S. D. (1982). Principles and practice in second language acquisition (1st ed). Pergamon.

Kuppens, A. H. (2010). Incidental foreign language acquisition from media exposure. Learning, Media and Technology, 35(1), 65-85. https://doi.org/10.1080/17439880903561876

Kusyk, M. et Sockett, G. (2012). From informal resource usage to incidental language acquisition: Language uptake from online television viewing in English. ASp. La Revue Du GERAS, 62, 45-65. https://doi.org/10.4000/asp.3104

Le Cor, G. (2001). La fiction à substrat professionnel comme médiation du milieu professionnel, étude de cas : The Hunt for Red October de Tom Clancy et son adaptation cinématographique. ASp, 31-33, 215-226. https://doi.org/10.4000/asp.1970

Lee, J. S. (s. d.). (2019). Quantity and diversity of informal digital learning of English. Language Learning, 23(1), 114-126.

Lindgren, E. et Muñoz, C. (2013). The influence of exposure, parents, and linguistic distance on young European learners' foreign language comprehension. International Journal of Multilingualism, 10(1), 105-129. https://doi.org/10.1080/14790718.2012.679275

MacIntyre, P. D., Clément, R., Dörnyei, Z. et Noels, K. A. (1998). Conceptualizing Willingness to Communicate in a L2: A Situational Model of L2 Confidence and Affiliation. The Modern Language Journal, 82(4), 545. https://doi.org/10.2307/330224

Mangenot, F. (2002). Forums et formation à distance: Une étude de cas. Éducation permanente, 152(3). https://hal.archives-ouvertes.fr/hal-02056899

Mangenot, F. et Penilla, F. (2009). Internet, tâches et vie réelle. Le français dans le monde, 45, 82-90.

Médioni, M.-A. (2009). L'enseignement-apprentissage des langues : Un agir ensemble qui s'affirme. Les langues modernes. http://www.aplv-languesmodernes.org/ 
Nissen, E. (2003). Apprendre une langue en ligne dans une perspective actionnelle : Effets de l'interaction sociale [thèse de doctorat, Université Strasbourg I - Louis Pasteur].

Nissen, E. (2011). Variations autour de la tâche dans l'enseignement / apprentissage des langues aujourd'hui. A/sic, 14. https://doi.org/10.4000/alsic.2344

Ollivier, C. (2012). Approche interactionnelle et didactique invisible - Deux concepts pour la conception et la mise en œuvre de tâches sur le web social. Alsic., 15(1). https://doi.org/10.4000/alsic.2402

Papi, C. (2016a). De l'évolution du métier d'enseignant à distance. Sciences et Technologies de l'Information et de la Communication pour l'Éducation et la Formation, 23(1), 15-45. https://doi.org/10.3406/stice.2016.1691

Papi, C. (2016b). L'université et son public entre traditions et évolutions. Questions de communication, 31, 249-269.

Peters, E. et Webb, S. (2018). Incidental vocabulary acquisition through viewing L2 television and factors that affect learning. Studies in Second Language Acquisition, 40(3), 551-577. https://doi.org/10.1017/S0272263117000407

Petit, M. (1999). La fiction à substrat professionnel: Une autre voie d'accès à l'anglais de spécialité. ASp, 23-26, 57-81. https://doi.org/10.4000/asp.2325

Petit, M. (2000). Le paratexte dans la fiction à substrat professionnel. Bulletin de la société de stylistique anglaise, 21, 173-195.

Petit, M. (2002). Éditorial. ASp. la revue du GERAS, 35-36, 2-3.

Petit, M. (2004). Quelques réflexions sur la fiction à substrat professionnel : Du général au particulier. Dans Aspects de la fiction à substrat professionnel (Petit, Michel, Isani, Shaeda, p. 3-23). Imprimerie de l'Université Victor Segalen Bordeaux 2.

Prabhu, N. S. (1987). Second Language Pedagogy. Oxford University Press.

Puimège, E. et Peters, E. (2019). Learning L2 vocabulary from audiovisual input: An exploratory study into incidental learning of single words and formulaic sequences. The Language Learning Journal, 47(4), 424-438. https://doi.org/10.1080/09571736.2019.1638630

Sarré, C. et Whyte, S. (2016). Research in ESP teaching and learning in French higher education: Developing the construct of ESP didactics. ASp, 69, 139-164. https://doi.org/10.4000/asp.4834

Schmitt, N. et Redwood, S. (2011). Learner knowledge of phrasal verbs: A corpus-informed study. Dans F. Meunier, S. De Cock, G. Gilquin et M. Paquot (dir.), A Taste for Corpora (p. 173-208). John Benjamins Publishing Company. https://www.jbe-platform.com/content/books/9789027287083-scl.45.12sch

Sockett, G. (2011). From the cultural hegemony of English to online informal learning: Cluster frequency as an indicator of relevance in authentic documents. ASp, 60, 5-20. https://doi.org/10.4000/asp.2469

Sockett, G. (2014). The Online Informal Learning of English. Palgrave Macmillan.

Sockett, G. et Kusyk, M. (2013). L'apprentissage informel en ligne : Nouvelle donne pour l'enseignement-apprentissage de l'anglais. Recherche et pratiques pédagogiques en langues de spécialité. Cahiers de l'APLIUTt, 32(1), 75-91. https://doi.org/10.4000/apliut.3578

Sotillo, S. M. (2000). Discourse Functions and Syntactic Complexity in Synchronous and Asynchronous Communication. Language Learning and Technology, 4(1), 77-110.

Springer, C. (2010). La dimension sociale dans le CECR: Pistes pour scénariser, évaluer et valoriser l'apprentissage collaboratif. Canadian Modern Language Review / La revue canadienne des langues vivantes, 66(4), 511-523. https://doi.org/10.3138/cmlr.66.4.511

Stevens, A. et Shield, L. (2010). Étude sur l'impact des technologies de l'information et de la communication (TIC) et des nouveaux médias sur l'apprentissage des langues. Commission Européenne. https://web2learn.eu/wp-content/uploads/2016/12/Final_Report_FR.pdf

Stockwell, G. (2010). Effects of Multimodality in Computer- Mediated Communication Tasks. Dans M. Thomas et H. Reinders (dir.), Task-Based Language Learning and Teaching with Technology (p. 83-104). London: Continuum.

Sundqvist, P. (2009). Extramural English Matters: Out-of-School English and Its Impact on Swedish Ninth Graders' Oral Proficiency and Vocabulary [Karlstad University]. https://www.skolporten.se/forskning/avhandling/extramuralenglish-matters-out-of-school-english-and-its-impact-on-swedish-ninth-graders-oral-proficiency-and-vocabularyl

Webb, S. (2015). Extensive Viewing: Language Learning through Watching Television. Dans D. Nunan et J. C. Richards (dir.), Language Learning Beyond the Classroom (p. 159-168). Routledge. https://ir.lib.uwo.ca/edupub/107 
Webb, S. et Rodgers, M. P. H. (2009a). Vocabulary Demands of Television Programs. Language Learning, 59(2), 335-366. https://doi.org/10.1111/j.1467-9922.2009.00509.x

Webb, S. et Rodgers, M. P. H. (2009b). The Lexical Coverage of Movies. Applied Linguistics, 30(3), $407-427$. https://doi.org/10.1093/applin/amp010 